\author{
Титова Анна Васильевна
}

\title{
ВЛИЯНИЕ ИНВЕСТИЦИОННОЙ ДЕЯТЕЛЬНОСТИ НА ФИНАНСОВУЮ УСТОЙЧИВОСТЬ ПРЕДПРИЯТИЯ
}

Финансовая стабильность означает, что финансовая система способна противостоять потрясениям в экономике и на финансовых рынках, выступать посредником в кредитах и платежсах и надлежацим образом перераспределять риски. B статье приведен анализ степени воздействия инвестиционной деятельности на финансовую устойчивость предприятия. Для достижсеия поставленной цели были проанализированы теоретическое, аналитическое и методическое обеспечение и эмпирический опьтт реализации инвестиционного проекта.

Ключевые слова: финансовая стабильность, инвестиционная деятельность, инвестиционный проект.

\section{Anna Titova \\ THE IMPACT OF INVESTMENT ACTIVITY ON THE FINANCIAL STABILITY OF THE ENTERPRISE}

Financial stability means that the financial system is equipped to withstand shocks to the economy and financial markets, to mediate credit and payments, and to redistribute risks appropriately. The analysis of the degree of impact of investment activities on the financial stability of the enterprise. To achieve this goal, an analysis of the theoretical, analytical, empirical and methodological support for the implementation of investment project was make.

Key words: financial stability, investment activities, investment project.

Bведение / Introduction. Любое предприятие в своей деятельности должно функционировать и развиваться. В процессе функционирования и развития предприятия особое значение приобретают понятия равновесия и устойчивости. Устойчивость - это способность системы сохранять текущее состояние при наличии внешних воздействий. Более устойчивым является такое предприятие, которое при равных внешних и внутренних воздействиях подвержено меньшим изменениям и отклонениям от прежнего состояния.

Одной из характеристик стабильного положения предприятия служит его финансовая устойчивость. Обеспечение финансовой устойчивости является типичной проблемой, с которой сталкивается каждый руководитель предприятия. Она зависит от стабильности экономической среды и от результатов его функционирования и эффективного реагирования на изменения факторов внутренней и внешней среды.

Финансовая устойчивость предприятия - это оценка рисков, которые связаны с финансовым обеспечением работы организации за счет источников средств, привлеченных со стороны. У каждого предприятия есть два источника финансирования: собственные средства и привлеченные средства. Собственным источником финансирования деятельности является кредит, предоставленный предприятию его владельцем на весь период осуществления деятельности. Получается, собственным источником финансирования будут средства, которые предприятие не возвращает обратно. У привлеченного же источника средств существуют точные сроки существования - до момента обязательного погашения кредиторской задолженности (возвращения кредитов).

Финансовую устойчивость определяют таюже мерой обеспечения предприятия необходимыми финансовыми ресурсами для осуществления деятельности и возможности своевременно исполнять свои обязательства. 
Важным направлением в деятельности предприятия является формирование и реализация инвестиционной политики. При принятии решений об инвестировании возникает такая проблема, как необходимость оценочной деятельности. Прежде чем принять решение, следует произвести анализ и оценку необходимости инвестиций.

Эффективность инвестиционного проекта дает инвестору необходимую информацию, которая будет использована при определении необходимости и выгоды инвестирования средств на текущий момент времени.

Maтериалы и методы / Materials and methods. Исследование в методологическом плане опирается на теоретические методы (анализ, синтез, конкретизация, обобщение, сравнение, метод аналогий), методы логического, системного и сравнительного анализа, динамические методы оценок, эмпирические экспериментальные (компьютерное моделирование), графическая визуализация процессов.

Результаты и обсуждение / Results and discussion. Рассмотрим проблему влияния инвестиционной деятельности на финансовую устойчивость предприятия на примере расчета эффективности инвестиционного проекта для предприятия ЗАО ПКФ «Севкавсвязьсервис». Для улучшения эффективности деятельности предприятия, предлагается инвестиционный проект по созданию сайта в сети Интернет.

Бизнес-сайт - это веб-сайт, на котором представлена информация о компании, а также о сфере ее деятельности. Это эффективный источник данных, где существует возможность заявить о компании, рассказать о ее преимуществах, видах деятельности, изменениях, результатах работы с клиентами, получать обратную связь в виде отзывов. Также это отличный способ рекламы для организации и положительная имиджевая составляющая, которая сформирует лояльное отношение потенциальных и укрепит такое отношение уже реальных клиентов.

Для определения уровня риска и целесообразности осуществления разработанного нами инвестиционного проекта следует провести оценку эффективности и результативности данного проекта. При инвестировании 3 млн руб. и сроке их действия в течение 3 лет прогнозируется получить годовые доходы P1 = 0,8 млн руб., $\mathrm{P} 2=1,3$ млн руб., P3 = 3,2 млн руб. Коэффициент r составит $12 \%$.

Расчет производится на основе данных статистики о среднем размере инфляции в $4 \%$ и повышении объьема выручки с учетом применения инвестиционного проекта предположительно на $3 \%$.

Оценка эффективности инвестиционного проекта по созданию сайта организации представлена в таблице 1.

Оценка эффективности инвестиционного проекта, тыс. руб.

Таблица 1

\begin{tabular}{|c|c|c|}
\hline Период & Без учета инвестиций & С учетом инвестиций \\
\hline А & 1 & 2 \\
\hline 2017 год & 2221,00 & 2287,63 \\
\hline 2018 год & 2309,84 & 2379,14 \\
\hline 2019 год & 2402,23 & 2474,30 \\
\hline
\end{tabular}

С привлечением инвестиций выручка в прогнозируемом периоде заметно увеличится, данные по динамике представлены на рисунке. 


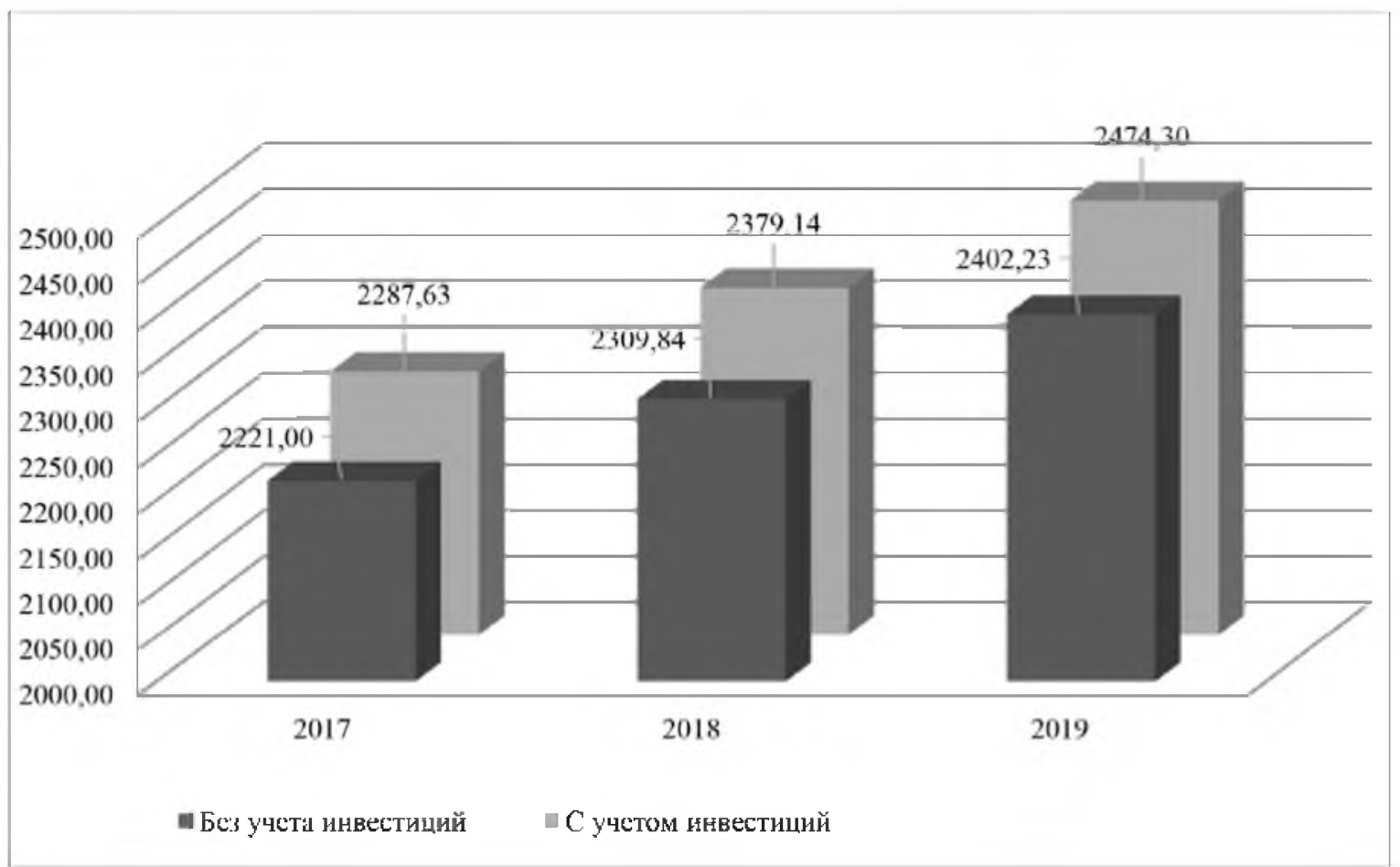

Рис. Динамика выручки в прогнозируемом периоде

Предполагаемая прибыль ЗАО ПКФ «Севкавсвязьсервис» в прогнозируемых периодах представлена в таблице 2 .

Таблица 2

Предполагаемая прибыль ЗАО ПКФ «Севкавсвязьсервис» за 2017-2019 гг., тыс. руб.

\begin{tabular}{|c|c|c|c|}
\hline Период & Без учета инвестиций & С учетом инвестиций & Инвестиционная прибыльь \\
\hline A & 1 & 2 & 3 \\
\hline 2017 & 152,00 & 952,00 & 800,00 \\
\hline 2018 & 158,08 & 1458,08 & 1300,00 \\
\hline 2019 & 164,40 & 3364,40 & 3200,00 \\
\hline
\end{tabular}

В результате применения данного проекта организация за тот же самый прогнозируемый период получает прибыль в разы больше, чем без использования проекта.

Проведем динамическую оценку эффективности проекта. Расчет чистой дисконтированной ценности является разницей между затратами на реализацию ивестиций и полученнной прибылью. Этот метод позволяет понять, какой доход принесет проект, окупится ли он в принципе или он убыточен, когда он сможет окупиться и сколько денег принесет в конкретный момент времени, зачастую используется для сравнения инвестиционных проектов.

$$
N P V=\sum_{i} \frac{C F t}{(1+i)^{t}}-I C,
$$

где $C F$ - сумма чистого денежного потока в период времени (месяц, квартал, год и т. д.); $\mathrm{t}$ - период времени, за который берется чистый денежный поток; $N$ - количество периодов, за который рассчитывается инвестиционный проект; $i$ - ставка дисконтирования; $I C$ - первоначальные вложения. 
Используя формулу, мы можем рассчитать величину дисконтированных доходов и чистый приведенный эффект рассматриваемого инвестиционного проекта.

$$
\begin{gathered}
P V=800 /(1+0,12) 1+1300 /(1+0,12) 2+3200 /(1+0,12) 3=4040,00, \\
\text { NPV }=4040,00-3000,00=1040,00, \\
1040,00>0
\end{gathered}
$$

следовательно, проект прибыльный и его следует принять к рассмотрению.

Индекс рентабельности инвестиций $(P I)$ - это показатель количества прибыли на единицу инвестиций. Индекс рентабельности инвестиций помогает оценить потенциал сделки перед заключением договора, отследить динамику роста или сокращения прибыли, посчитать, сколько всего денег принесло инвестирование после завершения проекта и вывода вложений. Индекс рентабельности инвестиций показывает целесообразность инвестиционного проекта: если $P I>1$, это значит, что проект рентабельный и его можно принять к рассмотрению; если $P I=1$, то проект следует проанализировать по другим показателям оценки эффективности инвестиционных вложений, если $P I<1$, то проект убыточен.

$$
P I=\sum_{i} \frac{P t}{(1+i)^{t}} / I C .
$$

Рассчитаем индекс рентабельности проекта:

$$
\begin{gathered}
P I=4040,00 / 3000,00=1,35 . \\
1,35>1,
\end{gathered}
$$

значит, проект рентабельный.

Внутренняя норма доходности (рентабельности) инвестиций (IRR) - это такая ставка дисконта, при которой нет убытков по вкладам, или предельное значение процента, при котором чистая приведённая стоимость будет нулевой. В зависимости от сравнения показателей $\mathrm{I} R R$, $N P V$ и $r$ (реальная ставка дохода в процентах), принимается решение о вложении в проект. Если $I R R>r, N P V>0$, то можно инвестировать, если $I R R=r, N P V=0$, то необходимо посчитать дополнительные показатели, если $I R R<r, N P V<0$, то инвестировать нельзя. Данный метод позволяет определить прибыльность возможного вложения (чем больше размер доходности, тем выше рентабельность вложений) и оптимальную ставку кредита, т. к. $I R R$ - это предельная цена безубыточности вложения.

$$
I R R=r_{1}+\frac{N V P\left(r_{1}\right)}{N V P\left(r_{1}\right)-N V P\left(r_{2}\right)} \cdot\left(r_{2}-r_{1}\right) .
$$

где $r$ - процентная ставка.

Вначале необходимо узнать минимальную IRR:

$$
I R R_{\min }=\sqrt[\sqrt{\sum_{k-1}^{k-N} F V_{k}}]{I C}-1
$$

где $N$ - число лет; $I C$ - первоначальная стоимость вклада; $\sum$ - сумма доходов за каждый год в тысячах рублей, все числа должны быть разделены на 1000.

Необходимо вычислить для каждого предельного значения показателя свой размер NPV:

$$
\begin{gathered}
N P V=-I C+\sum_{t=1}^{N} \frac{C F_{t}}{(1+I R R)^{i}} . \\
I R R_{\min }=\sqrt[3]{\frac{0,8+1,3+3,2}{3}}-1=0,21=21 \% .
\end{gathered}
$$




$$
I R R_{\max }=\frac{0,8+1,3+3,2}{3}-1=0,77=77 \% .
$$

Данные показатели говорят о том, что внутренняя норма доходности находится в промежутке между $21 \%$ и $77 \%$. Рассчитаем $N P V$ для предельных показателей:

$$
\begin{aligned}
& N P V(21 \%)=-3+\frac{0,8}{1,21}+\frac{1,3}{1,21^{2}}+\frac{3,2}{1,21^{3}}=0,36 \\
& N P V(77 \%)=-3+\frac{0,8}{1,77}+\frac{1,3}{1,77^{2}}+\frac{3,2}{1,77^{3}}=-1,55
\end{aligned}
$$

Можно рассчитать величину $I R R$ для данной инвестиции:

$$
I R R=0,21+\frac{0,36}{0,36-(-1,55)} \cdot(0,77-0,21)=0,32=32 \% .
$$

Расчет показал, что размер ставки дисконта сверх 32 \% будет убыточным для инвестиционного проекта. На данный момент проект является прибыльным, т. к. соблюдается неравенство $\operatorname{IRR}>r(32 \%>12 \%)$.

Дисконтированный период окупаемости инвестиций (DPP) учитывает стоимость денег во времени, в отличии от статического метода определения срока окупаемости инвестиций. $D P P=\min n$, при котором в случае дисконтирования срок окупаемости увеличивается, т. е. всегда будет верно неравенство $D P P>P P$.

$$
D P P=\sum_{i=1}^{n} \frac{C F_{i}}{(1=r)^{i}}>I C,
$$

где $I C$ - первоначальная стоимость вклада; $C F$ - сумма чистого денежного потока в период времени; $r$ - ставка дисконтирования; $\mathrm{n}$ - период реализации проекта.

Приведем денежные потоки в текущую стоимость:

$$
\begin{gathered}
P V_{1}=800 /(1+0,12)=714,29 \text { тыс. руб. } \\
P V_{2}=1300 /(1+0,12) 2=1040,00 \text { тыс. руб. } \\
P V_{3}=3200 /(1+0,12) 3=2269,50 \text { тыс. руб. }
\end{gathered}
$$

Определим период окупаемости инвестиций:

$$
714,29+1040,00+2269,50=4023,79 \text { тыс. руб. }
$$

Сумма доходов за первые два года меньше размера инвестиции в 3000,00 тыс. руб., следовательно, срок окупаемости больше двух лет. $(1-(4023,79-3000,00) / 2269,50)=0,55$ остаток третьего года.

В результате мы выяснили, что дисконтированный срок окупаемости проекта равен 2,55 года и все рассчитанные показатели определили прибыльность и рентабельность данного инвестиционного проекта.

Заключение / Conclusion. Устойчивость финансового состояния предприятия зависит от результатов его деятельности в области производства, коммерции и инвестиций. Из приведенных в работе расчетов основных показателей деятельности предприятия видно, что в данных условиях финансовое положение предприятия будет устойчиво. Инвестиционный проект обладает высокими показателями экономической эффективности и является выгодным вариантом вложения инвестиций, т. к. организация в прогнозируемом периоде получит дополнительную прибыль и вложения окупятся менее чем за три года. На основе данных, полученных в результате исследования по рассматриваемой проблеме, можно заключить, что инвестиционная деятельность и её грамотная оценка имеют непосредственное влияние на деятельность предприятия и его финан- 
совую устойчивость. Инвестиции имеют множество назначений, их размер зависит от масштабности инвестиционного проекта, а поскольку целью инвестирования всегда является получение прибыли или иных экономически выгодных результатов, то существует необходимость оценки целесообразности вложений и эффективности их использования.

\section{ЛИТЕРАТУРА И ИНТЕРНЕТ-РЕСУРСЫ}

1. Анофриков С. П. и др. Экономика: учебное пособие / С. П. Анофриков, Д. А. Казначеев, Н. В. Кулешова, А. Н. Савиных. М.: КноРус, 2016. 240 с.

2. Безденежных М. М., Севастьянова Н. Б. Экономика общественного сектора: учеб̆ое пособие. М.: КноРус, 2016. 208 c.

3. Борисовская Т. А., Ильчиков М. З. Микроэкономика. Макроэкономика: учебник/подобщ. ред. В. Д. Камаева. М.: КНОРУ.С, 2016. 384 с.

4. Грязнова А. Г. Микроэкономика. Практический подход (Managerial Economics): учебник. М.: КноPyc, 2017. $682 \mathrm{c}$.

5. Дмитриев Ю. А., Васильева Л. П. Региональная экономика: учебник. М.: КноРус, 2015. 264 с.

6. Карагезян У. В. Неконкурентное поведение в российской хозяйственной практике: мотивы фирм и последствия для экономики // Экономический анализ: теория и практика. 2015. № 14. С. 25-34.

7. Демодоран А. Инвестиционная оценка: Инструменты и техника оценки любых активов / пер. с англ. 5-е изд. М.: Альпина Бизнес Букс, 2008. 1340 с.

8. Мирская С. Ю., Ливанцов И. С. Выбор оптимальной концепции по структурированию данных для систематизации бизнес-процессов // Наука и образование: хозяйство и экономика; предпринимательство; право и управление. 2017. № 6 (85). С. 40-45.

\section{REFERENCES AND INTERNET RESOURCES}

1. Anofrikov S. P. Ekonomika (Economics): uchebnoe posobie / S. P. Anofrikov, D. A. Kaznacheev, N. V. Kuleshova, A. N. Savinykh. M.: KnoRus, 2016. 240 s.

2. Bezdenezhnykh M. M., Sevast'yanova N. B. Ekonomika obshchestvennogo sektora (Economics of the public sector): uchebnoe posobie. M.: KnoRus, 2016, 208 s.

3. Borisovskaya T. A., Il'chikov M. Z. Mikroekonomika, Makroekonomika (Microeconomics, macroeconomics): uchebnik / pod obshch. red. V. D. Kamaeva. M.: KNORUS, 2016. 384 s.

4. Gryaznova A. G. Mikroekonomika, Prakticheskii podkhod (Managerial Economics): uchebnik. M.: KnoRus, 2017. $682 \mathrm{~s}$.

5. Dmitriev Yu. A., Vasil'eva L. P. Regional'naya ekonomika (Regional economy): uchebnik. M.: KnoRus, 2015. $264 \mathrm{~s}$.

6. Karagezyan U. V. Nekonkurentnoe povedenie $\mathrm{v}$ rossiiskoi khozyaistvennoi praktike: motivy firm i posledstviya dlya ekonomiki (Anti-competitive behavior in the Russian business practice: motives of firms and the consequences for the economy) // Ekonomicheskii analiz: teoriya i praktika. 2015. No. 14. S. 25-34.

7. Demodoran A. Investitsionnaya otsenka: Instrumenty i tekhnika otsenki lyubykh aktivov (Investment appraisal: Tools and techniques for valuing any assets) / per. s angl. 5-e izd. M.: Al'pina Biznes Buks, 2008. $1340 \mathrm{~s}$.

8. Mirskaya S. YU., Livancov I. S. Vybor optimal'noj koncepcii po strukturirovaniyu dannyh dlya sistematizacii biznes-processov (The choice of the optimal concept of data structuring for systematization of business processes) // Nauka i obrazovanie: hozyajstvo i ehkonomika; predprinimatel'stvo, pravo i upravlenie. 2017, No. $6(85)$. S. $40-45$.

\section{СВЕДЕНИЯ ОБ АВТОРЕ}

Tитова Анна Васильевна, магистрант, Ставропольский институт кооперации (филиал) БУКЭП, г. Ставрополь. E-mail: anna.titova.indi@mail.ru

\section{INFORMATION ABOUT AUTHOR}

Anna Titova, graduate student, Stavropol Institute of cooperation (branch) Belgorod University of cooperation, Economics and law, Stavropol. E-mail: anna.titova.indi@mail.ru 